\title{
Guide to tracks \& signs of wildlife: An update
}

\section{BOOK TITLE:}

A field guide to the tracks and signs of southern, Central and East African wildlife

\section{AUTHORS: \\ Chris Stuart}

Mathilde Stuart

ISBN:

9781770073609

\section{PUBLISHER:}

Struik Nature, Cape Town, R320

\section{PUBLISHED:}

\section{3}

\section{REVIEW TITLE:}

Guide to tracks \& signs of wildlife: An update

\section{REVIEWER:}

Charlene Bissett

\section{AFFILIATION:}

Department of Zoology and Entomology, Rhodes University, Grahamstown, South Africa

\section{EMAIL:}

c.bissett@ru.ac.za

\section{POSTAL ADDRESS:}

Department of Zoology and

Entomology, Rhodes University, P0 Box 94, Grahamstown 6140, South Africa

\section{HOW TO CITE:}

Bissett C. Guide to tracks \& signs of wildlife: An update. S Afr J Sci. 2013;109(7/8), Art. \#a0025, 1 page. http://dx.doi. org/10.1590/sajs.2013/a0025
Being able to interpret tracks and signs in the bush is an important skill for wildlife enthusiasts, researchers, field guides, game rangers and trackers and can aid any nature lover in interpreting a story that unfolded in the bush.

Chris and Mathilde Stuart have over 30 years experience in wildlife research and book writing and together they have worked in the field of biodiversity survey and research and photography in 50 different countries. They have published many scientific and popular articles and 18 books, including several best-selling field guides such as the Field Guide to Mammals of Southern Africa. Their field guides are comprehensive and regularly revised and updated to ensure that the latest biological and ecological information is covered.

The latest revised field guide - A Field Guide to the Tracks and Signs of Southern and East African Wildlife was originally published in 1994 and quickly became the standard reference to the subject in the region and was reprinted many times. This new edition not only covers detail of tracks, but also includes detail on droppings, bird pellets, nests and shelters, and feeding signs for mammals, birds, reptiles, amphibians and insects. This new edition has been greatly expanded to include new features such as full-colour photographs of the animal species to supplement the extensive examples of tracks and signs, additional quick reference keys and coverage of central African species.

The introduction includes pointers on where to look for tracks and signs, as well as how to interpret, measure, collect and preserve these tracks and signs. The addition of a ruler on the back page helps with measuring tracks and other signs while in the field. This source is valuable for researchers that are studying elusive animal species and need to rely on tracks and signs to interpret the behaviour and abundance of their study animals. It also allows for standardised methods of measuring track size and stride length, ensuring that research projects are comparable.

The chapters have been divided into easy to follow sections such as 'tracks', 'droppings' and 'feeding signs' and the contents page is detailed, ensuring easy access to a particular chapter. In addition to the quick reference keys on the insides of the front and back covers of the book there are detailed reference keys in each chapter allowing readers to navigate quickly to the relevant sections of interest.

The authors have grouped tracks that you are likely to encounter into headings such as 'Paws' (cheetahs, lions etc.), 'Heavyweights' (rhinos) and 'Three toes' (aardvark). For each animal, photographs of the animals as well as of actual tracks and drawings of the 'perfect' track, including size measurements, have added great value to this book. In real life, animal spoor is likely to be smudged or indistinct and therefore the addition of photos of actual tracks showing these deviations from the 'perfect' track helps with the final identification of the tracks.

This new edition retains the unique approach that made the first edition so popular and will make an invaluable addition to the book collection of all those interested in wildlife. It is also available as an ebook in pdf and epub formats. 